10.2478/AMB-2020-0042

\title{
ADDITIVE ANTIDEPRESSANT EFFECTS OF COMBINED ADMINISTRATION OF ECITALOPRAM AND CALORIC RESTRICTION IN LPS-INDUCED NEONATAL MODEL OF DEPRESSION IN RATS
}

\author{
E. Haritov, J. Tivcheva \\ Department of Pharmacology and Toxicology, Faculty of Medicine, Medical University - Sofia, Bulgaria
}

\begin{abstract}
Background and aims: Increasing evidence indicates that inflammation in the periphery and neuroinflammation in the brain might be involved in the pathophysiology of depressive symptoms in humans. Relatively little is known about the effects of selective serotonin re-uptake inhibitors (SSRI) on individuals exposed to differential dietary regimens, like caloric restriction (CR). The aim of the current study is to assess the antidepressant and antineuroinflammatory effects of CR in single administration and combined with SSRIsantidepressant escitalopram in LPS-induced model of depression in Wistar rats. Materials and methods: For this purpose, we used 36 Wistar rats and applied 3 behavioral tests for depression (FST, SPT and NSFT) in animals and an ELISA-method for measurement of brain IL-1beta levels. Results: Behavioral assessment and results from ELISA-method have shown that $C R$ not only augments the effect of the antidepressant escitalopram on forced swim test (FST) and sucrose preference test (SPT), but also reduces the brain levels of proinflammatory cytokine IL-1beta. Combined with escitalopram, CR enhances antidepressant and antinflamatory properties of this SSRI. Discussion and conclusion: These results show that the response to antidepressive treatment depends on the diverse dietary regimens, especially low-caloric diet. We suggest that the background of this is augmentation of anidepressant and antineuronflammatory properties of some antidepressants by CR. Manipulation of dietary regimens is attractive and new strategy for the management of pharmacoresistant depression.
\end{abstract}

Key words: cytokines, depression, environmental enrichment, rats, SSR/s

Corresponding author: Evgeni Haritov, MD, PhD, Department of Pharmacology and Toxicology, Faculty of Medicine, Medical University - Sofia, 2 Zdrave Str., 1431 Sofia, Bulgaria, e-mail: evgeniharitov@gmail.com

\section{INTRODUCTION}

D epression is a common psychiatric disorder affecting a human's wellbeing and causing long term absence of work as well as mental and physical impairment [1]. There are numerous theories explaining the etiology of depression. A famous one is the presence of "biochemical disbal- ance" in the CNS [2]. Recent research into the matter shows that the depressive state has complex causes including other neurobiological mechanisms such as: disbalance in the CNS neurotrophic elements and neuroinflamation [3]. The need of new pathogenetic mechanisms is obvious and these would explain problems related to: 1 . Discovery of new neurobiological targets for antidepressive drugs; 2 . Identifi- 
cation of depression-related biomarkers for patients responding to the therapy versus non-responders (patient monitoring). One promising perspective in this regard is unveiling the role of neuroinflammation in depression development.

Recent research shows, that physiological stress contributes in the depressive state trigger and this is mediated via the neuroinflammation. Animal models with an application of lipopolysaccharide (LPS) show an increase in the levels of pro-inflammatory cytokines, such as TNF-alfa and IL-1 beta. This represents an established experimental model of depression [4]. Symptoms that are observed in animals are similar to those encountered in humans [5].

Current antidepressive drugs acting on monoamine neurotransmission are effective, however in over $30 \%$ of patients, clinical remission is not achieved, despite numerous treatments [6]. Selective serotonin re-uptake inhibitors (SSRIs) are preferred anti-depressive drugs because they are well - tolerated and safe. In the last decade, a great deal of scientific evidence has shown that part of the therapeutic potential of antidepressants is likely to be due to their suppressive effect on neuroimmune alterations, associated with affective disorders. Clinical trials show, however, that half of all patients don't show an adequate clinical response to the respective drug. Or alternatively, a so called approach for amplifying the effect of the first administered antidepressant in the vast majority of patients is attempted [7]. Unfortunately, even these additional strategies do not guarantee complete remission. The need of complimentary non-pharmacological therapeutic strategies that need to be justified from a clinical point of view is obvious.

Caloric restriction (CR), defined as a dietary regimen low in total calories with maintenance of the essential composition of the diet at a normal level, in the interest of avoiding malnutrition [8], is a potent intervention which increases both average and maximum lifespan, retards physiological signs of aging, and decreases incidence and severity of age-related diseases, such as neoplasias, kidney disease, diabetes, in a variety of model organisms [9].

A number of studies proposed a variety of molecular mechanisms for how CR exerts beneficial effects, for example, reduction of inflammatory response, up-regulation of endogenous and exogenous antioxidants and changes in apoptosis [10]. In the nervous system, CR regimen has been shown to be neuroprotective. Principally, $\mathrm{CR}$ delays neuroinflammation associated with aging [11], attenuates pro-inflammatory pathways and enhances anti-inflammatory pathways in the hippocampus [12].
Despite the long history of investigations on caloric restriction $(C R)$ in medicine, its role in the treatment of mental disorders has not been studied. Many theories have been proposed regarding the mechanisms by which CR exerts its therapeutic effects, but so far the exact and cell-molecular mechanism has not been resolved [13]. Considering the above facts about the role of neuroinflammation in the pathogenesis of affective disorders and the need of new therapeutic approaches for improving the effects of antidepressants, the aim of the current trial was: 1) to investigate the effects of single and combined applications of Escitalopram and caloric restriction of the behavioral tests for depression; 2) to assess the effects of single and combined applications of Escitalopram and caloric restriction to neuroinflammation.

\section{MATERIALS AND METHODS}

\section{Drugs, tests and models:}

> Escitalopram (Lundbek, Denmark, $10 \mathrm{mg} / \mathrm{kg}$, per os). The drug is dissolved in saline $(0.9 \% \mathrm{NaCl}$ solution) and introduced via intragastral tube.

> LPS (lipopolysaccharide)-Escherichia coli, serotype 055: B5 (Sigma, $50 \mathrm{mcg} / \mathrm{kg}$, i.p.).

$>$ ELISA method (kit BioVendor) assessing IL-1 beta is used for reading the pro-inflammatory cytokines levels in brain homogenate.

$>$ To assess the influence of the CR, special type of CR-regimen was applied. Daily caloric intake of some experimental groups of animals was reduced by $40 \%$ for 4 weeks.

$>$ Forced swim test (FST)

$>$ Sucrose preference test (SPT)

> Novelty-suppressed feeding test (NSFT)

\section{Experimental animals}

Thirty six (male gender) neonatal rats from breed Vistar were used from the Central vivarium, MU - Sofia. They were previously split into groups (six rats per group $n=6$ ). Ten days after delivery (post-natal day 10), the respective animals (groups 2, 4, 5 and 6 ) were taken out of the cages for a limited period of time. They were weighed and treated with LPS once only $(50 \mathrm{mcg} / \mathrm{kg})$. After this standard procedure they were returned to the cages with their respective dams up till postnatal day 21 .

The neonatal male rats (body weight from 30 to $50 \mathrm{~g}$ ) were split from their dams on postnatal day 21 and divided into 6 groups, 6 rats each. They were variously treated with LPS on postnatal day 10. Groups 3,4 and 6 (CR-groups) received $60 \%$ of the amount 
consumed by groups 1 and 2 ad libitum groups-AL) for a period of 4 weeks. Constant room temperature of $21 \mathrm{C}+/-2 \mathrm{C}$ with alternating light/ dark (ratio 12:12 h) environment reflecting the circadian rhythm of the animals was assured. Food and water were available ad libitum. The experiments were performed between $8.00 \mathrm{am}-15.00 \mathrm{pm}$. They were conducted in compliance with the requirements of the Medical University's Ethic Comity - Sofia.

\section{EXPERIMENTAL DESIGN}

The 21 day old post-natal rats were divided into 6 groups (6 animals in each $(n=6))$. These groups were as follow:

Group 1: rats were treated with saline $(0.9 \% \mathrm{Nacl})$, per os for 3 weeks, commencing on post-natal day 21 (after the animals were split form the mothers). This group was used for negative control.

Group 2: Positive control group 1 (LPS group) was treated once only with LPS $(50 \mathrm{mcg} / \mathrm{kg}$ on post-natal day 10 , i.p.).

Group 3: Positive control group 2 (CR 60\% group) received $60 \%$ of the amount consumed by groups $A L$, after post-natal day 21, for four weeks.

Group 4: Experimental group 1 (LPS and CR 60\% - group) was treated only once on post-natal day 10 with LPS (50 mcg/kg), i.p. and after post-natal day 21 received $60 \%$ of the amount consumed by groups $A L$, for four weeks.

Group 5: Experimental group 2 (LPS + Escitalopram group) was treated once only with LPS (50 $\mathrm{mcg} / \mathrm{kg}$ ) on post-natal day 10 . Sequentially on postnatal day 21 (after splitting from their dams) the rats were given Escitalopram (10 mg/kg, per os) for a period of three weeks.

Group 6: Experimental group 3 (LPC + Escitalopram+ CR 60\%) was treated once with LPC (50 mcg/ $\mathrm{kg}$ ), i.p. on post-natal day 10 . Sequentially on postnatal day 21 (after splitting from their mothers) the rats were given a combination of Escitalopram (10 $\mathrm{mg} / \mathrm{kg}$, per os) for a period of three weeks and simultaneously received $60 \%$ of the amount consumed by groups $A L$, for four weeks.

After the 4 week period completion, behavioral tests for depression were undertaken by the animals (Forced swim test-FST, Sucrose preference test SPT and novelty suppressed feeding test-NSFT). Immediately after the test completion the rats were decapitated, and homogenate made of the removed brains. The sandwich ELISA method was used for reading of IL-1 proinflammatory levels.

\section{DIETARY REGIMEN}

A regular regimen was applied for CR [14]. Both, control and $C R$ rats received the same laboratory pellets. Rats in groups $A L$ (1, 2 and 5$)$ were fed ad libitum $(A L)$, and the daily food ingestion was constant across the experimental period (25-35 g/day). Rats in Groups CR (3, 4 and 6 ) received $60 \%$ of the quantity of food consumed by the rats in Groups AL. To avoid undernourishment, vitamin and mineral mixtures were added to the diet in Groups CR to make the dietary micronutrient content equal to that in Groups $A L$; however, the final caloric content of the diet in Groups CR was $40 \%$ lower than that in Groups AL. Food was weighed and provided to the animals in Groups CR daily, approximately $1 \mathrm{~h}$ before the start of the dark cycle. The dietary regimens were started after 1 week of adaptation and continued during the experimental period.

\section{BEHAVIORAL TESTS FOR DEPRESSION}

After a period of 4 weeks with CR-administration and 3 weeks with escitalopram, the animals were subjected to 3 behavioral tests characterizing depression levels (FST, SPT and NSFT):

\section{Forced swim test (FST)}

The test is used to assess the depressive symptoms in the experimental animals. It consists of two sessions, $15 \mathrm{~min}$ and $5 \mathrm{~min}$ respectively over a 24 hour period. The experiment tool is a cylinder filled with $40 \mathrm{~cm}$ room temperature water. Within 5 min intervals the following parameters are assessed (indicators for presence or absence of depressive symptoms): 1) Immobility time; 2) Swimming time; 3) Active climbing on the walls of the cylinder.

An extended period of immobility is considered as a sign of a depressive state in the experimental animals.

\section{Sucrose preference test (SPT)}

Decreased consumption of sucrose is an equivalent of anhedonia in depressive patients in clinical practice. Within 24h duration, animals in the cages are supplied with two bottles, one with water and one with sucrose. The locations of the bottles are exchanged in the middle of the experiment. The so called index of sucrose consumption is calculated as a ratio of:

Quantity of consumed sucrose $(\mathrm{ml}) /$ quantity of consumed sucrose $(\mathrm{ml})+$ quantity of consumed water $(\mathrm{ml})$.

\section{Novelty-suppressed feeding test}

The NSFT test is often used as a tool to assess depressive behavior in experimental animals. Similar to the "open field test", the NSFT test is based on the innate 
fear of open spaces in rodents. In addition, the NSFT test introduces an additional component of motivation, with the pursuit of animal nutrition, in the absence of food, conflicting with the fear of new open spaces. This gives the NSFT test excellent predictive validity for the antidepressant efficacy of a drug or approach.

The test is briefly conducted as follows: In the center of a white plastic box $(50 \times 50 \times 40 \mathrm{~cm})$, place a single food pellet of ordinary rodent food. After deprivation of food for 24 hours, the rats were placed individually in the corner of the plastic box. The time after which the rats begin to chew the food pellets is reported as a latency of feeding in a new environment. Antidepressant pretreatment has been found to reduce this latency. The box is cleaned and the food pellet is replaced after each animal.

\section{Brain tissue collection and IL-1 reading in hippo- campus}

Animals were decapitated, brains immediately removed and hippocampuses separated. Subsequently these were homogenized using a protein extraction reagent, containing a protease inhibitor. Probes were centrifuged $15000 \mathrm{~g} / 10 \mathrm{~min}$, and the derived supernatant used for IL-1 beta reading by ELISA (BioVendor - detection reference $=4,8 \mathrm{pg} / \mathrm{ml}$; detection range $=12.5-800 \mathrm{pg} / \mathrm{ml}$ ), accordingly to the manufacturer's instructions. Absorption was determined for $450 \mathrm{~nm}$ and $620 \mathrm{~nm}$ by the ELISA reader.

\section{STATISTICAL ANALYSIS}

Data processing was done by means of variance analysis-One-Way ANOWA. For each criterion/indicator mean arithmetic values (Mean) and standard deviations (+/-SEM) were given. A statistically significant difference $p<0.05$ is accepted.

\section{RESULTS}

\section{Behavioral tests}

Forced swim test (FST)

The duration of the immobility time in the experimental groups is shown in Fig. 1. A statistically significant difference between group 2 with LPS administration and control group was logically established $\left({ }^{* * *} p<\right.$ $0.001)$. On the other hand, administration of escitalopram and KD in groups 4 and 5, respectively, resulted in a statistically significant reduction in the immobility period in these groups compared to group 2 with LPS $\left({ }^{*} p<0.05\right)$. Of particular importance is the fact that the combined administration of escitalopram and KD in the last 6th experimental group showed statistically significant differences $\left({ }^{* *} p<0.01\right)$, compared to the groups (4th and 5th) with self-administered escitalopram and CD.

\section{Sucrose preference test (SPT)}

The sucrose preference test, that is an indicator for anhedonia in animals, showed similar trends. The analysis data presented in Fig. 2 reveals statistically significant differences in the sucrose preference test as follows: Animals in Group 2 have the lowest sucrose consumption compared to the other groups, and in particular the control group $\left({ }^{* *} p<0.001\right)$. Pre-treatment of animals with escitalopram in group 4 and access to KD in group 5 resulted in significantly increased sucrose intake and a difference ( $\left.{ }^{*} p<0.05\right)$ compared with the LPS group. The highest level of sucrose consumption similar to FST was found in the combination group of escitalopram and KD $\left({ }^{* *} \mathrm{p}<\right.$ 0.01 ) compared to the groups (4th and 5 th) with selfadministered escitalopram and KD.

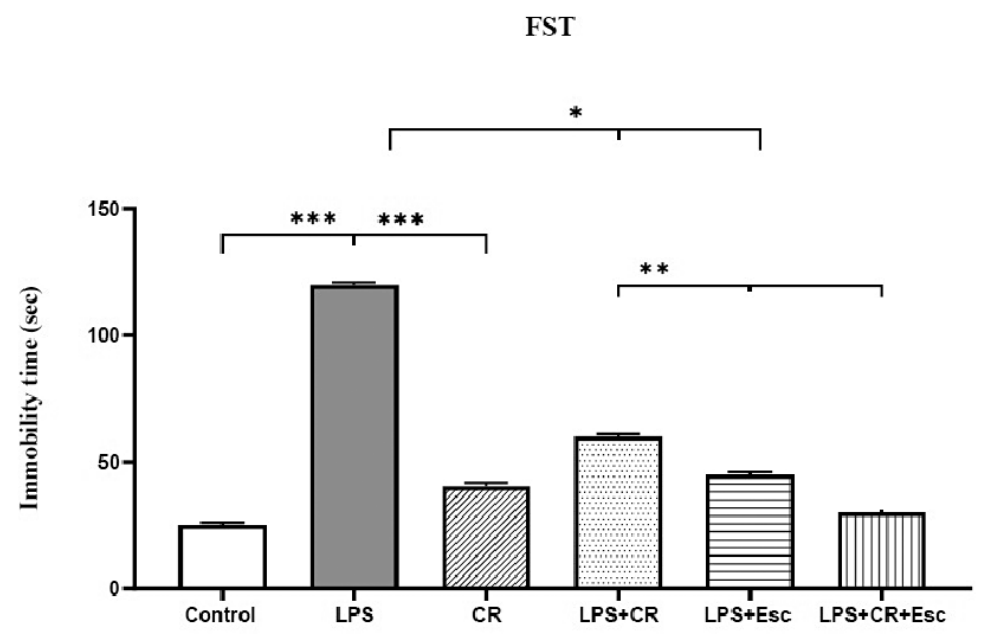

Fig. 1. Effects of single and combined administration of escitalopram and CR $60 \%$ on FST (immobility time). All data are represented as mean $\pm \operatorname{SEM}\left(n=6\right.$ animals per group). ${ }^{*} p<0.05 ;{ }^{* *} p<0.01 ;{ }^{* * *} p<0.001$ 


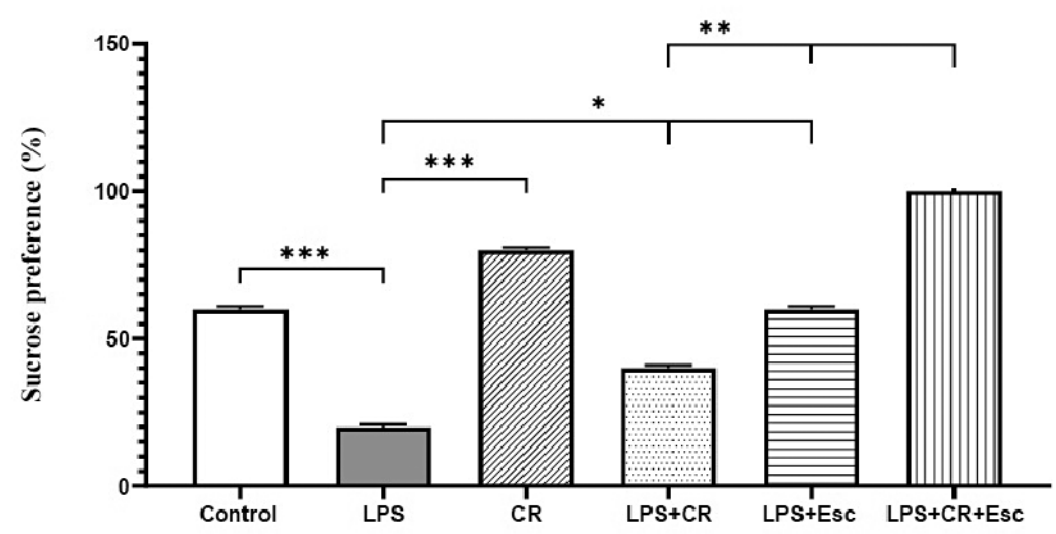

Fig. 2. Effects of single and combined administration of escitalopram and CR $60 \%$ on SPT. All data are represented as mean \pm SEM $(n=$ 6 animals per group). ${ }^{*} p<0.05 ;{ }^{* \star} p<0.01 ;{ }^{* \star *} p<0.001$

\section{Novelty-suppressed feeding test (NSFT)}

In the NSFT test, the data of which is presented in Fig. 3, there are similar trends as in the previous two behavioral tests. Again, attention is drawn to data revealing a statistically significant difference $\left({ }^{*} p<0.05\right)$ between groups 4 and 5 , on the one hand, and the 6 th on the other, in which there is a combined administration of escitalopram and KD.

\section{ELISA method for proinflammatory cytokine IL-1 beta levels in brain homogenate}

Our results with the sandwich-ELISA of brain homogenate, revealed the same findings (Fig. 4) as those in FST and SPT and NSFT. The statistically significant reduction of IL-1beta readings in group 6, compared to group 4 and 5 (with individual application of CR $60 \%$ or Escitalopram) was confirmed $\left({ }^{* *} p<0.01\right)$.

\section{DISCUSSION}

Depression is a widespread psychiatric condition, with recurring episodes of the illness, and sometimes fatal outcomes. The pathogenesis is still under scientific research, and recently (last 10 years) the role of the disturbed immune mechanisms (CNS) as a trigger and factor in disease progression has been emphasized. Data taken from metanalysis [15] shows increased blood levels of the proinflammatory cytokines TNF-al-

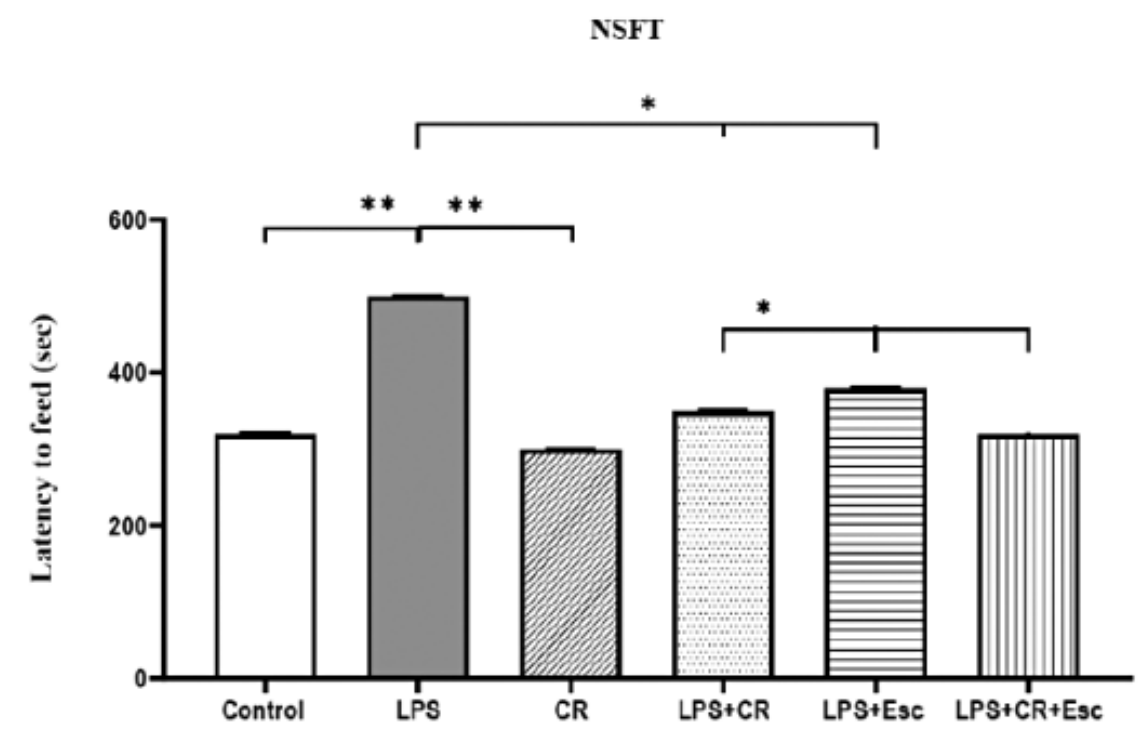

Fig. 3. Effects of single and combined administration of escitalopram and CR $60 \%$ in the NSFT-test. All data are represented as mean \pm SEM ( $n=6$ animals per group). ${ }^{*} p<0.05 ;{ }^{* *} p<0.01 ;{ }^{* * *} p<0.001$ 


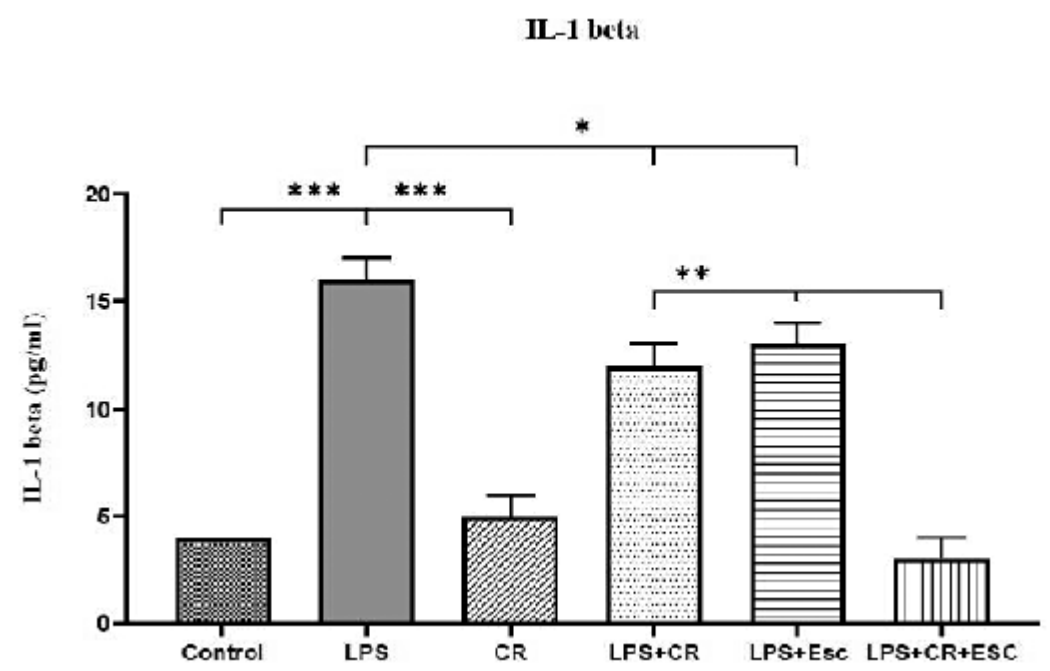

Fig. 4. Effects of single and combined administration of escitalopram and CR $60 \%$ on IL-1beta levels in the hippocampus. All data are represented as mean \pm SEM ( $n=6$ animals per group). ${ }^{*} p<0.05 ;{ }^{* *} p<0.01 ;{ }^{* * *} p<0.001$

fa and IL-1 beta in patients with depression who do not take antidepressants, in comparison with healthy individuals. The postmortem brain examination of such patients showed also an increase in the gene expression of proinflammatory cytokines [16].

The hypothesis for neuroimmune disturbance as a key factor in the depressive progression is supported by a well-known fact: Some NSAIDs (Celecoxib) act synergistically with the selective serotonin reuptake inhibitors (SSRI) [17]. These and other facts clearly support the hypothesis that central and peripheral inflammatory mechanisms exist and their suppression would induce a therapeutic response. Given the fact that the application of antidepressants is related to drug resistance in $30-40 \%$ of patients, the need for alternative therapeutic approaches is obvious.

The search for new therapeutic alternatives in the light of the above facts also draws attention to the application and exploration of the antidepressant potential of alternative methods, such as some dietary strategies. One of them is calorie restriction, investigated and applied in various fields of medicine [18]. For the first time, the data from this study suggest that part of the effects of CR related to the depressive symptomatic response may be correlated with a reduction in the levels of proinflammatory cytokines in the CNS and an enhancement by this mechanism of the SSRIs-antidepressant effects.

Experimental animal models show that the intake of bacterial endotoxin lipopolysaccharide (LPS) does induce depressive symptoms in respective tests in rodents, such as the forced swim test (FST), etc [19]. Regarding the use of SSRI drug escitalopram, the in vitro and in vivo experiments shows antiinflammatory properties [20]. A wide range of health benefits of CR have been identified in animal and human studies [21]. In contrast, no studies assessing the effect of $C R$ in neuroinflammatory alterations induced by LPS administration have been conducted to date. Based on studies showing that CR can protect neurons against degeneration in animal models of injury that engage inflammatory processes (stroke and seizures) [22], we asked whether CR might modify the effects of systemic inflammation on depressive symptoms and activity of escitalopram. Using LPS to cause an immune response and depressive symptoms, we observed significant worsening of depressive parameters in FST, SPT and NSFT, which were counteracted by CR. Our paper has established that LPS administration leads to a significant increase in the levels of IL-1 beta in the hypoccampuses of the experimental animals. Conversley an application of escitalopram after postnatal day 21 leads to significantly reduced readings for IL-1 beta.

Of particular importance are our data showing that in the groups with combined administration of calorie restriction and escitalopram (group 6), the most pronounced antidepressant effect was observed in the three behavioral tests (FST, SPT, NSFT). On the other hand, these data correlate with the similar in the ELISA-method for the levels of IL-1beta.

Previous studies indicated that $\mathrm{CR}$ attenuates neuroinflammation through up-regulating silent information regulator 1 (SIRT1) in hippocampus [23]. SIRT1 deacetylates nuclear factor kappa B (NF-kB), and inhibits its activity, which facilitates the synthesis and release of a wide cascade of cytokines, including IL-1 beta and TNF-alfa. The transcription of NLRP3, which 
plays a crucial role in releasing the precursor pro-IL1 beta to its mature form, is also dependent on the activation of NF-KB [24]. Therefore, the results from our paper, in combination with previous studies, could be suficient to draw the conclusion that $\mathrm{CR}$ reduced depressive symptoms and reinforced the antidepressant efficacy of escitalopram, most likely via inhibiting neuroinflammation. Our results may have implications for the development of new therapeutic strategies in antidepressive therapy. The data presented here give new insights into the therapeutic and antidepressant effect of the low-calorie dietary regimens. Aditional neuroprotective mechanisms associated with neuroimmune activity of CR are yet to be discovered.

\section{CONCLUSION}

In conclusion, the data from the present study warrant future studies and the introduction of $C R$ as an additive antidepressant tool, potentiating the effects of modern antidepressants. This would provide new direction in explaining the mechanism of CR, linking its metabolic effects with various neuroimmune substrates in the CNS. Clinically validating synergistic effects with antidepressants would turn CR into an important means of overcoming pharmacoresistance in the treatment of depression and would decipher the deep molecular mechanisms of affective disorders linking neurometabolism to impaired CNS immune regulation in various mental illnesses.

Acknowledgement: This work is supported by the Bulgarian Ministry of Education and Science under the National Program for Research "Young Scientists and Postdoctoral Students".

Disclosure summary: The authors have nothing to disclose.

\section{REFERENCES}

1. Zorilla EP, Luborsky L, McKay JR et al. The relationship of depression and stressors to immunological assays: A metaanalytic review. Brain Behav Immun. 2001; 15: 199-226.

2. Rush AJ. STAR*D: What have we learned. Am J Psychiatry. 2007; 164: 739-752.

3. Antonioli M, Rybka J, Carvalho LA. Neuroimmune endocrine effects of antidepressants. Neuropsychiatr Dis Treat. 2012; 8: 65-83.

4. Wu CW, Chen YC, Yu L et al. Treadmill exercise counteracts the suppressive effects of peripheral lipopolysaccharide on hippocampal neurogenesis and learning and memory. J Neurochem. 2007; 103: 2471-2481.

5. Miller AH, Maletic $\mathrm{V}$, Raison $\mathrm{CL}$. Inflammation and its discontents: the role of cytokines in the pathophysiology of major depression. Biol Psychiatry. 2009; 65(9): 732-741.
6. Musselman DL, Lawson DH, Gumnick JF et al. Paroxetine for the prevention of depression induced by high-dose interferon alfa. N Engl J Med. 2001; 344: 961-966.

7. Hwang J, Zheng LT, Ock J et al. Inhibition of glial inflammatory activation and neurotoxicity by tricyclic antidepressants. Neuropharmacology. 2008; 55(5): 826-834.

8. Koubova J, Guarente L. How does calorie restriction work? Genes Dev. 2003; 17, 313-21.

9. Mattison JA et al. Impact of caloric restriction on health and survival in rhesus monkeys from the NIA study. Nature. 2012; 489, 318-21.

10. Lopez-Lluch $\mathrm{G}$ et al. Calorie restriction induces mitochondrial biogenesis and bioenergetic efficiency. Proc Natl Acad Sci USA. 2006; 103: 1768-73.

11. Lee CK, Weindruch R, Prolla TA. Gene-expression profile of the ageing brain in mice. Nat Genet. 2000; 25: 294-7.

12. Monti B, Virgili M, Contestabile A. Alterations of markers related to synaptic function in aging rat brain, in normal conditions or under conditions of long-term dietary manipulation. Neurochem Int. 2004; 44: 579-84.

13. Fusco $S$ et al. A role for neuronal cAMP responsive-element binding (CREB)-1 in brain responses to calorie restriction. Proc Natl Acad Sci USA. 2012; 109: 621-6.

14. Pugh TD, Klopp RG, Weindruch R. Controlling caloric consumption: protocols for rodents and rhesus monkeys. Neurobiol Aging. 1999; 20: 157-65.

15. Dowlati $\mathrm{Y}$, Herrmann N, Swardfager W et al. A meta-analysis of cytokines in major depression. Biol Psychiatry. 2010; 67(5): 446-457.

16. Wong ML, Dong C, Maestre-Mesa J. Polymorphisms in inflammation-related genes are associated with susceptibility to major depression and antidepressant response. Mol Psychiatry. 2008; 13(8): 800-812.

17. Akhondzadeh $\mathrm{S}$, Jafari $\mathrm{S}$, Raisi $\mathrm{F}$ et al. Clinical trial of adjunctive celecoxib treatment in patients with major depression: a double blind and placebo controlled trial. Depression Anxiety. 2009; 26(7): 607-611.

18. Martin CK, Bhapkar M, Pittas AG et al. Effect of calorie restriction on mood, quality of life, sleep, and sexual function in healthy nonobese adults: the CALERIE 2 randomized clinical trial. JAMA Intern Med. 2016; 1189:1-10.

19. O Conor J, Castanon $\mathrm{N}$ et al. Lipopolysaccharide-induced depressive-like behavior is mediated by indoleamine 2,3-dioxygenase activation in mice. Mol Psychiatry. 2009; 14(5): 511-522.

20. Horikawa H, Kato TA, Mizoguchi $Y$ et al. Inhibitory effects of SSRIs on IFN-gamma induced microglial activation through the regulation of intracellular calcium. Prog Neuropsychopharmacol Biol Psychiatry. 2010; 34(7): 1306-1316.

21. Mattson MP, Wan R. Beneficial effects of intermittent fasting and caloric restriction on the cardiovascular and cerebrovascular systems. J Nutr Biochem. 2005; 16:129-137.

22. Bruce-Keller AJ, Umberger G, McFall R. Food restriction reduces brain damage and improves behavioral outcome following excitotoxic and metabolic insults. Ann Neurol 1999; 45:8-15.

23. Chen $D$ et al. The role of calorie restriction and SIRT1 in prion-mediated neurodegeneration. Exp Gerontol. 2008; 43: 1086-93.

24. Latz E, Xiao TS, Stutz A. Activation and regulation of the inflammasomes. Nat Rev Immunol. 2013; 13: 397-411.

Received: 20 July 2019, Accepted: 20 August 2019 